\title{
Dynamic Functional Connectivity and Symptoms of Parkinson's Disease: A Resting-State fMRI Study
}

\author{
Gwenda Engels ${ }^{1 *}$, Annemarie Vlaar ${ }^{2}$, Brónagh McCoy ${ }^{3}$, Erik Scherder ${ }^{1}$ and Linda Douw ${ }^{4,5}$ \\ ${ }^{1}$ Department of Clinical, Neuro and Developmental Psychology, Faculty of Behavior and Movement Sciences, VU University, \\ Amsterdam, Netherlands, '2Department of Neurology, Onze Lieve Vrouwe Gasthuis (OLVG), Amsterdam, Netherlands, \\ ${ }^{3}$ Department of Experimental and Applied Psychology \& Institute of Brain and Behavior, Faculty of Behavior and Movement \\ Sciences, VU University, Amsterdam, Netherlands, ${ }^{4}$ Department of Anatomy and Neurosciences, VU University Medical \\ Center, Amsterdam, Netherlands, ${ }^{5}$ Department of Radiology, Athinoula A. Martinos Center for Biomedical Imaging, \\ Massachusetts General Hospital, Charlestown, MA, United States
}

OPEN ACCESS

Edited by:

Panteleimon Giannakopoulos, Université de Genève, Switzerland

Reviewed by: Julien Bogousslavsky, Swiss Medical Network, Switzerland Rongfeng Qi, Medical School of Nanjing University,

China

*Correspondence: Gwenda Engels gwenda.engels@gmail.com

Received: 23 May 2018 Accepted: 05 November 2018 Published: 23 November 2018

Citation:

Engels G, Vlaar A, McCoy B, Scherder E, and Douw $L$ (2018) Dynamic Functional Connectivity and Symptoms of Parkinson's Disease: A Resting-State fMRI Study.

Front. Aging Neurosci. 10:388. doi: 10.3389/fnagi.2018.00388
Research has shown that dynamic functional connectivity (dFC) in Parkinson's disease $(\mathrm{PD})$ is associated with better attention performance and with motor symptom severity. In the current study, we aimed to investigate dFC of both the default mode network (DMN) and the frontoparietal network (FPN) as neural correlates of cognitive functioning in patients with $\mathrm{PD}$. Additionally, we investigated pain and motor problems as symptoms of PD in relation to dFC. Twenty-four PD patients and 27 healthy controls participated in this study. Memory and executive functioning were assessed with neuropsychological tests. Pain was assessed with the Numeric Rating Scale (NRS); motor symptom severity was assessed with the Unified Parkinson's Disease Rating Scale (UPDRS). All subjects underwent resting-state functional magnetic resonance imaging (fMRI), from which dFC was defined by calculating the variability of functional connectivity over a number of sliding windows within each scan. dFC of both the DMN and FPN with the rest of the brain was calculated. Patients performed worse on tests of visuospatial memory, verbal memory and working memory. No difference existed between groups regarding $\mathrm{dFC}$ of the DMN nor the FPN with the rest of the brain. A positive correlation existed between $\mathrm{dFC}$ of the DMN and visuospatial memory. Our results suggest that dynamics during the resting state are a neural correlate of visuospatial memory in PD patients. Furthermore, we suggest that brain dynamics of the DMN, as measured with dFC, could be a phenomenon specifically linked to cognitive functioning in $\mathrm{PD}$, but not to other symptoms.

Keywords: dynamic functional connectivity, default mode network, frontoparietal network, Parkinson's disease, cognitive impairment, pain, motor symptoms

\section{INTRODUCTION}

The organization of structural and functional connections of the brain, or brain topology, shapes complex behavior (Park and Friston, 2013; Stam, 2014): cognitive functioning arises from both local and global integration across the whole brain. Local integration, which mainly entails short-range neural connections, subserves specialized cognitive functions. Global integration entails long-range neural connections, and underlies higher cognitive functions (Park and Friston, 2013; Sporns, 2013). Cognitive dysfunctioning has been related to altered brain network topology as measured 
by resting-state imaging in several disorders, such as in multiple sclerosis (Hawellek et al., 2011; Meijer et al., 2017), schizophrenia (Lynall et al., 2010) and epilepsy (Douw et al., 2011).

Parkinson's disease (PD) is a neurodegenerative disorder, hallmarked by motor symptoms: rigidity, bradykinesia, tremor and postural instability. Although clinical diagnosis of PD is based on these motor symptoms, non-motor symptoms play an equally, sometimes even more devastating role in patients' quality of life (Martinez-Martin et al., 2011). One such non-motor symptom is cognitive decline, e.g., in the executive and memory domains (Litvan et al., 2012). In line with the abovementioned link between network integration and cognition, cognitive decline in PD has been associated with decreased functional connectivity (Olde Dubbelink et al., 2014; Amboni et al., 2015) or deviant coupling between subnetworks of the brain (Putcha et al., 2016). Another non-motor symptom is pain (Broen et al., 2012; Choi et al., 2017), a symptom that is present in about two thirds of patients and has been associated with sleep and mood disturbances, as well as severity of motor symptoms (Broen et al., 2012; Defazio et al., 2017). Pain in PD has been linked to a functional disconnection between the hippocampus and nucleus accumbens (Polli et al., 2016).

Connectivity analyses on resting-state functional magnetic resonance imaging ( $\mathrm{rs}-\mathrm{fMRI}$ ) have, until recently, been based on the assumption that connections remain stable throughout an fMRI session. However, temporal fluctuations of connectivity may in fact be a fundamental feature of brain networks, particularly in the context of cognitive functioning (Sizemore and Bassett, 2017). Dynamic functional connectivity (dFC) is an approach to assess these temporal fluctuations, by calculating the variability of functional connectivity over a number of sliding windows within each scan. $\mathrm{dFC}$ may have added value in explaining cognitive functioning above and beyond that of static connectivity, as it may detect the brain network's ability to deal with varying demands from the environment. Higher dFC (throughout the brain) has been related to better performance in healthy controls in the domains of cognitive flexibility, sustained attention and working memory (Jia et al., 2014). Also, lower dFC of a hub region of the default mode network $(\mathrm{DMN})$, the posterior cingulate cortex (PCC), was associated with worse memory performance in epilepsy patients, suggesting that high $\mathrm{dFC}$ is favorable in the memory domain (Douw et al., 2015).

The DMN is a task-negative network (i.e., deactivated during cognitive processes) that appears to be particularly vulnerable to the effects of disease (Broyd et al., 2009). Dynamics of the DMN appear to be associated with internallyoriented cognition (Kucyi and Davis, 2014). Another network that is involved in cognitive functioning is the frontoparietal network (FPN), which plays a central role in flexibly adapting to the environment (Cole et al., 2013). Dynamics of the FPN have been implicated in cognitive control (Zanto and Gazzaley, 2013). In healthy controls, a lower resting-state dFC between FPN and the DMN was related to a higher cognitive flexibility (Douw et al., 2016). Regarding cognitive functioning in $\mathrm{PD}$, a higher $\mathrm{dFC}$ of the dorsal attentional network at rest appears to be beneficial for performance on an attention task (Madhyastha et al., 2015a,b). Although $\mathrm{dFC}$ has been suggested to be associated with mild cognitive impairment (MCI) before (Jones et al., 2012), specific cognitive domains have not yet been investigated with respect to $\mathrm{dFC}$ in PD.

The relationship between $\mathrm{dFC}$ and other symptoms of $\mathrm{PD}$, such as pain or motor symptom severity, has been largely underexplored. One study investigated dynamics of the resting state in $\mathrm{PD}$ by determining brain states with a sliding window technique. Two brain states were found: one relatively more integrated and one more segregated brain state. Time spent in the more integrated brain state was correlated with the severity of motor symptoms of PD patients (Kim et al., 2017). Pain in general, i.e., not specifically related to $\mathrm{PD}$, may also depend on dynamic properties of the brain (Kucyi et al., 2013; Kucyi and Davis, 2015). However, this association seems primarily related to the modulation of pain by the cognitive domain of attention, instead of pain itself: a higher tendency to attend to painful stimuli was related to higher dFC.

This study investigates behavioral correlates of brain dynamics during the resting-state in $\mathrm{PD}$. The primary aim is to investigate whether $\mathrm{dFC}$ of the DMN and the FPN is a correlate of cognitive functioning. More specifically, we hypothesize that $\mathrm{dFC}$ of the DMN with the rest of the brain shows an association with memory functioning and that $\mathrm{dFC}$ of the FPN with the rest of the brain shows an association with executive functioning. We will further investigate $\mathrm{dFC}$ in $\mathrm{PD}$ by assessing a possible difference between PD patients with MCI and those who have intact cognitive functioning. In addition to cognition, we will investigate whether $\mathrm{dFC}$ relates to motor problems and pain in PD patients.

\section{MATERIALS AND METHODS}

\section{Subjects}

Patients were referred through neurologists of outpatient clinics (VU University Medical Center, Amsterdam, Netherlands; OLVG Hospital, Amsterdam, Netherlands; Zaans Medical Center, Zaandam, Netherlands). Healthy controls were recruited through advertisement in local newspapers, online advertisement and through participating patients (e.g., spouses, relatives, etc.). Inclusion criteria were: (1) age 40-75 years old; (2) ability to provide written informed consent; (3) normal or correctedto-normal vision; and (4) for patients only, a diagnosis of PD following UK Brain Bank criteria. Exclusion criteria for all subjects were: (1) current use of psychotropic medication other than levodopa, dopamine-agonists or other Parkinson medication; (2) major somatic disorder; (3) current psychiatric diagnosis as established by a psychiatrist and (4) presence of dementia, history of stroke or other neurological diseases (as stated in their medical status). A screening for dementia was performed using the Montreal Cognitive Assessment (MoCA; Nasreddine et al., 2005), with a cutoff of 21 or lower for dementia according to Biundo et al. (2014). Additionally, Beck's Depression Inventory (BDI) was administered to assess presence 
of depressive symptoms (Beck et al., 1996). Patients and controls were matched for age and sex. Education was categorized according to the Verhage-system, which runs from 1 (unfinished lower education) to 7 (finished university level; Verhage, 1964).

The study was carried out in accordance with regulations of the medical ethical committee of the VU University Medical Center (Amsterdam, Netherlands), who also approved the protocol. All subjects gave written informed consent in accordance with the Declaration of Helsinki. All methods were carried out in accordance with relevant guidelines and regulations.

\section{Procedure}

This project was part of a larger study investigating reinforcement learning, visual attention, and pain in PD. Sample sizes of 24 patients and 24 controls were pre-determined for the reinforcement learning task, however for the current study we could include additional subjects who passed the initial screening procedure (see "Results" section). Patients visited the hospital three times and controls twice. On the first day, all subjects underwent clinical assessment including neuropsychological testing, practiced a reward-learning task and filled out questionnaires.

Patients underwent MRI twice: once with their normal Parkinson medication ( $\mathrm{ON}$ phase), once without Parkinson medication (OFF phase). Only OFF phase imaging was used for this study in order to reduce the effect of dopaminergic medication. The OFF phase was defined as at least $12 \mathrm{~h}$ of dopaminergic medication withdrawal (overnight). One patient took their medication $8.5 \mathrm{~h}$ before the resting-state scan to relieve motor symptoms. Controls underwent MRI once. Note that only those procedures concerning the current project will be described in the remainder of the text. The MRI was generally planned in the same week as the clinical assessment, but no later than 60 days after clinical assessment.

\section{Clinical Assessment}

\section{Cognition, Motor Symptoms and Pain}

Since neuropsychological testing was performed on a different day than the scanning day, patients took their medication as normal, thus cognition was not tested during the OFF phase.

\section{Executive Functioning}

The Stroop Color Word test was used to investigate interference (Stroop, 1935). The interference measure was calculated by dividing the time needed for Stroop, card II (colored rectangles) by time needed for Stroop, card III (names of colors written in incongruent colors; Lansbergen et al., 2007), with a high score indicating a low degree of interference. To test verbal fluency, subjects were asked to generate as many words as possible from a specific semantic category within $1 \mathrm{~min}$, which was repeated with a different category (categories were first animals, then professions). Subjects were then asked to generate as many words as possible starting with a specific letter, which was repeated twice (letters were D, A and T). Word fluency was the average of both the category and the letter fluency tests. The Digit Span Backwards from the Wechsler Adult Intelligence Scale was administered to test working memory (Wechsler, 1955). Subjects were asked to repeat a series of digits in a reversed order. The number of correctly repeated series was used as working memory measure. The Rule-Shift Cards Test of the Behavioral Assessment of the Dysexecutive Syndrome (BADS) was used to assess mental flexibility (Wilson et al., 1998). Subjects were asked to respond to a set of stimulus cards according to a rule. In the second condition, the rule was changed. Number of mistakes was used to calculate a profile score, which served as a measure for mental flexibility.

\section{Memory}

Verbal memory was assessed using the Rey Auditory Verbal Learning Test (AVLT). The Dutch version of this test encompasses a list of 15 unrelated words (Saan and Deelman, 1986). In the immediate recall condition, the total number of correct words after five trials served as a measure for short-term verbal memory. In the delayed recall condition, the number of correct words after an interval of at least 15 min was used as a measure for long-term verbal memory. The Complex Figure of Rey (CFR) was used as an indication of visuospatial memory. Subjects were asked to copy a complex figure. After several minutes, subjects were asked to reproduce the figure without the original (Osterrieth, 1941). Scoring was performed according to a standardized method.

\section{Motor Functioning}

The motor part of the Unified Parkinson's Disease Rating Scale (UPDRS III) was administered during patients' OFF phase before MRI (Postuma et al., 2015). UPDRS III was always performed by the same person (GE).

\section{Pain Experience}

The Numeric Rating Scale (NRS) was administered before commencing the resting-state scan during the OFF phase. Subjects were asked to rate their pain on a scale from 0-10 for intensity of pain, with 0 indicating "no pain," and 10 indicating "the worst pain ever experienced."

\section{MRI}

Imaging data were collected with a 3T GE Signa HDxT MRI scanner (General Electric, Milwaukee, WI, USA) at the VU University Medical Center (Amsterdam, Netherlands). Structural images were acquired with a 3D T1-weighted magnetization prepared rapid gradient echo (MPRAGE) sequence with the following acquisition parameters: voxel size $=1 \mathrm{~mm}$ isotropic, 176 slices, $256 \times 256$ matrix, repetition time $(\mathrm{TR})=8.2 \mathrm{~ms}$, echo time $(\mathrm{TE})=3.2 \mathrm{~ms}$, flip angle $(\mathrm{FA})=12$ degrees, inversion time $(\mathrm{TI})=450 \mathrm{~ms}$. Restingstate data were acquired using a $\mathrm{T} 2 *$-weighted echo-planar functional scan: number of volumes $=202,42$ slices, slice thickness $=3.2 \mathrm{~mm}$, matrix size $=64 \times 64, \mathrm{TR}=2150 \mathrm{~ms}$, $\mathrm{TE}=35 \mathrm{~ms}, \mathrm{FA}=80$ degrees, field of view $(\mathrm{FOV})=240 \mathrm{~mm}$, total duration 7:12 min. For the resting-state scan, subjects were instructed to close their eyes, lie still and avoid falling asleep. The subject's head was immobilized using foam pads to reduce motion artifacts. 


\section{Processing of fMRI Data}

Data were analyzed using FSL FMRIB software library v5.0.9 (Jenkinson et al., 2012) and custom built scripts in bash and Matlab, version 2015a (Mathworks, Natick, MA, USA). The following pre-processing steps were taken: (1) images were corrected for head motion using MCFLIRT (Jenkinson et al., 2012); (2) bottom-up slice-timing correction was applied; (3) non-brain tissue was removed (using Brain Extraction Tool, BET); (4) functional images were registered to subject-space (T1-weighted structural image) using BBR; (5) the T1-weighted structural image was registered to MNI152 standard space (FLIRT for linear registration with 12 DOF); (6) high-pass filtering above $0.01 \mathrm{~Hz}$ was applied; (7) spatial smoothing was performed at $5 \mathrm{~mm}$ full-width half maximum (FWHM); (8) segmentation of gray and white matter was performed using FAST and SIENAX; and (9) the first three volumes of each resting-state scan were discarded to achieve field equilibrium.

\section{Dynamic Functional Connectivity}

See Figure 1 for a schematic overview of calculation of dFC. Time series of 264 atlas regions were used for subsequent analyses, based on the parcellation suggested by Power et al. (2011). These time series were scrubbed for motion outliers: time points with framewise displacement $>1.5 \mathrm{~mm}$ ( six DOF) were excluded from further analyses. Pearson's correlation coefficients were calculated between remaining time series from all regions of interest (ROIs) of the Power atlas, resulting in a $264 \times 264$ matrix per window per subject, with a window length of $60.2 \mathrm{~s}(28 \times \mathrm{TR})$ and a shift of $10.75 \mathrm{~s}(5 \times \mathrm{TR})$, resulting in 34 sliding windows per subject. The choice of window length was based on earlier studies (e.g., van Geest et al., 2018). The standard deviation for each connection was calculated and normalized for the average of that individual connection, yielding the coefficient of variation of each connection. This resulted in a separate dFC matrix per individual. Subsequently, we looked at dFC between the regions of the DMN (see Power et al., 2011 for included ROIs) with the rest of the brain, and at $\mathrm{dFC}$ between regions of the FPN (see Power et al., 2011 for included ROIs) with the rest of the brain.

\section{Statistical Analyses}

Statistical analyses were performed in IBM SPSS version 23 (Chicago, IL, USA). Normality of all variables was assessed with Kolmogorov-Smirnov tests and histogram inspection. Cognitive test scores were normally distributed, thus independent samples $t$-tests were performed for all cognitive measures, and False Discovery Rate (FDR) correction for multiple comparisons was applied with $Q<0.05$ (Hochberg and Benjamini, 1995). dFC measures were not normally distributed, thus differences in $\mathrm{dFC}$ measures were tested using non-parametric tests (MannWhitney). To test the association between dFC and cognition, a hierarchical linear regression using a stepwise forward method was performed per cognitive outcome measure that was significantly different between patients and controls. dFC of the DMN was used as a predictor for memory tests. dFC

A L
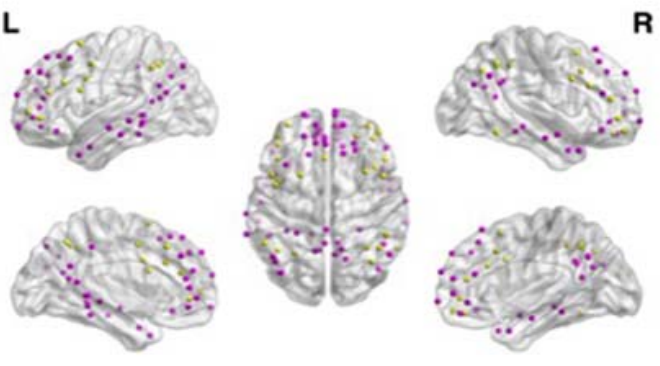

$\mathbf{R}$

$=\mathrm{DMN}$

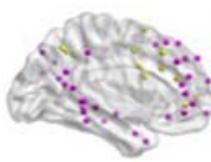

$\theta=$ FPN

B
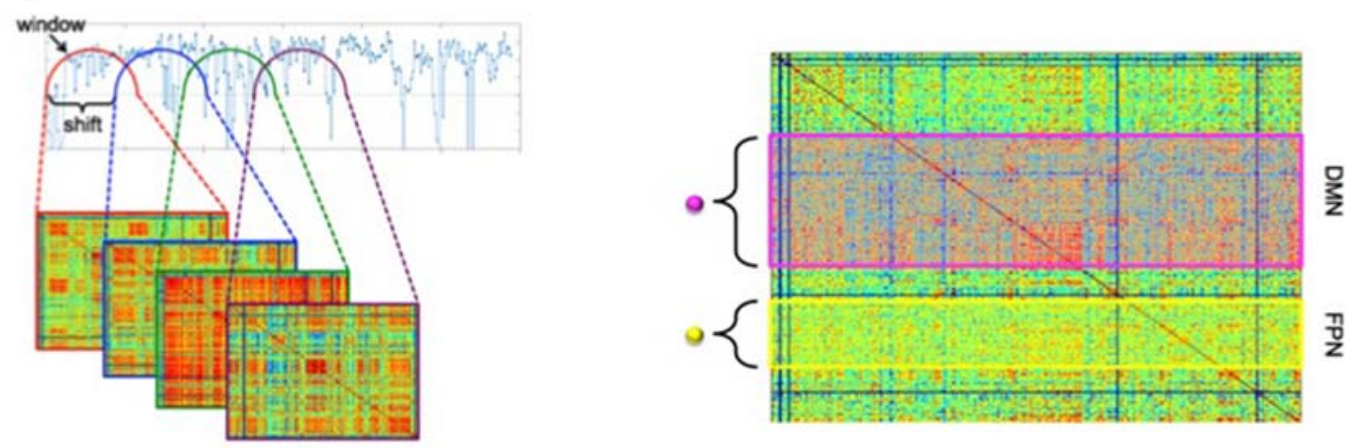

FIGURE 1 | Graphical representation of the calculation of dynamic functional connectivity (dFC). (A) Nodes of the default mode network (DMN) are depicted in pink; nodes of the frontoparietal network (FPN) are depicted in yellow. See Power et al. (2011) for specification of the networks. (B) A shifting window approach was used to calculate dFC. The left panel shows that an adjacency matrix was calculated for each shifted window. The standard deviation was then calculated for each connection and normalized for its average strength. The right panel of (B) shows a matrix of the resulting coefficients of variation for a single subject. Subsequently, an average was calculated for the DMN with the rest of the brain (pink rectangle in right panel) and for the FPN with the rest of the brain (yellow rectangle). Panel (A) was visualized using BrainNet Viewer (Xia et al., 2013). 
of the FPN was used as a predictor for tests of executive functioning. Separate hierarchical linear regression analyses were also performed with pain and motor symptoms as dependent variables, and $\mathrm{dFC}$ of the DMN and FPN as predictors. Residuals were normally distributed for all regression analyses, justifying the use of these parametric tests. In all regression analyses, average motion during the scan (as a variable) was added in the first step to control for a possible confounding effect of motion despite scrubbing high motion data points. Because of a possible relationship between cognitive decline and dynamics and because differences in static functional connectivity have been found in PD-MCI has been found before on measures of static functional connectivity (Baggio et al., 2015), we divided the group of patients into those with MCI and those without MCI, based on patients' score on the MoCA. We applied a cutoff score of $\leq 25$ as indicative of MCI (Hoops et al., 2009). Considering the explorative nature of this study, the association between PD symptoms and $\mathrm{dFC}$ was tested with an alpha level set at 0.05 , without correcting for multiple comparisons.

\section{DATA AVAILABILITY}

The datasets generated and analyzed during the current study are available from the corresponding author on reasonable request.

\section{RESULTS}

\section{Subjects}

Twenty-four patients and 27 healthy controls participated. See Figure 2 for a flowchart of in- and exclusion of patients. Characteristics of all subjects are shown in Table 1. Patients had a lower level of education than healthy controls $(U=174.50$, $p=0.003)$. Though not significantly different, patients had a lower score on the MoCA $\left(t_{(49)}=1.9, p=0.063\right)$. Patients had a higher score on the BDI, indicating more severe symptoms of depression in patients than in controls $\left(t_{(48)}=-5.107\right.$, $p<0.01)$.

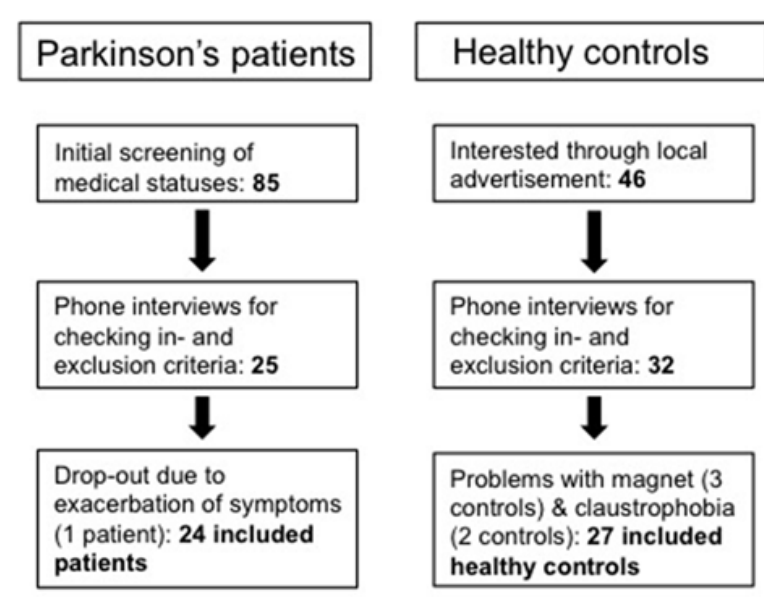

FIGURE 2 | Overview of in- and exclusion process for subjects.
TABLE 1 | Subject characteristics.

\begin{tabular}{|c|c|c|c|}
\hline & $\begin{array}{l}\text { Controls } \\
(n=27)\end{array}$ & $\begin{array}{l}\text { Patients } \\
(n=24)\end{array}$ & Statistics \\
\hline Age in years $(M, S D)$ & $59.37(8.54)$ & $63.42(7.93)$ & $\begin{aligned} t_{(49)} & =-1.749 \\
p & =0.087\end{aligned}$ \\
\hline $\begin{array}{l}\text { Education level according } \\
\text { to the Verhage-system } \\
\text { (Mdn, range) }\end{array}$ & $6(3)$ & $5(4)$ & $\begin{array}{l}U=174.50 \\
p=0.003\end{array}$ \\
\hline Sex & 11 females & 7 females & $\begin{aligned} x_{(1)}^{2} & =0.745 \\
p & =0.558\end{aligned}$ \\
\hline $\begin{array}{l}\text { Disease duration in years } \\
(\mathrm{M}, S D)\end{array}$ & - & $4.08(3.13)$ & - \\
\hline LEDD in $\mathrm{mg}(M, S D)^{\star}$ & - & $796.29(616.44)$ & - \\
\hline $\begin{array}{l}\text { UPDRS during OFF phase } \\
(\mathrm{M}, \mathrm{SD})\end{array}$ & - & 21.08 (8.31) & - \\
\hline $\operatorname{MoCA}(M, S D)$ & $27.89(1.89)$ & $26.88(1.92)$ & $\begin{aligned} t_{(49)} & =1.90 \\
p & =0.063\end{aligned}$ \\
\hline $\mathrm{BDI}$ & $22.96(2.24)$ & $30.46(7.12)$ & $\begin{aligned} t_{(48)} & =-4.938 \\
p & <0.001\end{aligned}$ \\
\hline
\end{tabular}

LEDD, Levodopa Equivalent Daily Dose; MoCA, Montreal Cognitive Assessment; BDI, Beck's Depression Inventory. *Twelve patients received Levodopa monotherapy, all other patients were taking a combination of Levodopa with DA-agonists or MAO-B/COMTinhibitors.

\section{Cognitive Performance}

Group differences in cognitive performance are summarized in Table 2. Patients performed worse on the CFR, and on both immediate and delayed recall of the AVLT. Fewer words were named in the verbal fluency tests by PD patients when compared to healthy controls. Interference score for the Stroop was similar for PD patients as for controls. No difference was found for number of digits correctly repeated backwards between PD patients and controls. No difference was found on performance on the BADS between PD patients and controls.

\section{Dynamic Functional Connectivity}

No difference was found between controls $(M d n=0.572)$ and patients $(M d n=0.941)$ regarding $\mathrm{dFC}$ between the DMN and the rest of the brain $(U=288.00, p=0.497)$. No difference was found between controls $(M d n=0.896)$ and patients $(M d n=0.962)$ regarding $\mathrm{dFC}$ between the FPN and the rest of the brain $(U=318.00, p=0.910)$.

\section{Association Between Dynamic Functional Connectivity and Cognitive Performance}

As described above, performance on the AVLT (both immediate and delayed recall), CFR and verbal fluency was lower in patients compared to controls (see Table 2). With respect to memory functioning, dFC of the DMN was positively associated with performance on the CFR $\left(R^{2}=0.219, F_{(1,21)}=5.979, p=0.023\right)$. $\mathrm{dFC}$ of the DMN was not associated with score on the immediate recall, or with score on the delayed recall of the AVLT. With respect to executive functioning, no association was between the dFC of the FPN and performance on verbal fluency tasks. See Table 3 and Figure 3 for details.

Additionally, we investigated $\mathrm{dFC}$ differences according to presence of MCI in PD patients. PD patients without MCI had a higher $\mathrm{dFC}$ of the DMN with the rest of the brain $(U=23.00$, $p=0.027)$. There was no difference in $\mathrm{dFC}$ of the FPN with the rest of the brain with respect to MCI $(U=46.00, p=0.535)$. 
TABLE 2 | Group differences in cognitive performance.

\begin{tabular}{|c|c|c|c|c|}
\hline & Controls $M(S D)$ & Patients $M(S D)$ & Statistics & $p$-value \\
\hline CFR & $24.37(4.98)$ & $18.56(7.32)$ & $t_{(45)}=3.17$ & $0.003^{*}$ \\
\hline AVLT immediate recall & $44.48(11.48)$ & $37.33(8.28)$ & $t_{(49)}=2.52$ & $0.015^{*}$ \\
\hline AVLT delayed recall & $9.26(3.60)$ & $6.88(2.54)$ & $t_{(46.72)}=2.75$ & $0.008^{*}$ \\
\hline BADS rule shift & $32.44(10.00)$ & $37.04(13.61)$ & $U=273.5$ & 0.402 \\
\hline Digitspan backwards & $6.85(2.10)$ & $6.33(2.33)$ & $t_{(49)}=0.841$ & 0.405 \\
\hline Stroop (interference) & $0.66(0.10)$ & $0.62(0.11)$ & $t_{(49)}=1.22$ & 0.227 \\
\hline
\end{tabular}

AVLT, Auditory Verbal Learning Test; BADS, Behavioral Assessment of the Dysexecutive Syndrome; CFR, Complex Figure of Rey. *Significant, corrected using the False Discovery Rate.

TABLE 3 | Association between dynamic functional connectivity (dFC) and cognitive functioning.

\begin{tabular}{llccccc}
\hline Network & Cognitive measure & Unstandardized B & Std. error of B & Standardized Beta & $\boldsymbol{p}$-value & Effect size $\left(\boldsymbol{R}^{2}\right.$ change $)$ \\
\hline DMN & CFR & 0.974 & 0.398 & 0.502 & $0.023^{*}$ & 0.219 \\
& AVLT immediate & 0.464 & 0.491 & 0.212 & 0.356 & 0.039 \\
& AVLT delayed & 0.214 & 0.151 & 0.317 & 0.171 & 0.039 \\
FPN & Verbal fluency & 0.405 & 0.267 & 0.305 & 0.145 & 0.083
\end{tabular}

Linear hierarchical regression analyses were performed with a forward stepwise method, per cognitive outcome measure. DMN, Default Mode Network; FPN, Frontoparietal Network; CFR, Complex Figure of Rey; AVLT, Auditory Verbal Learning Test; * significant association.

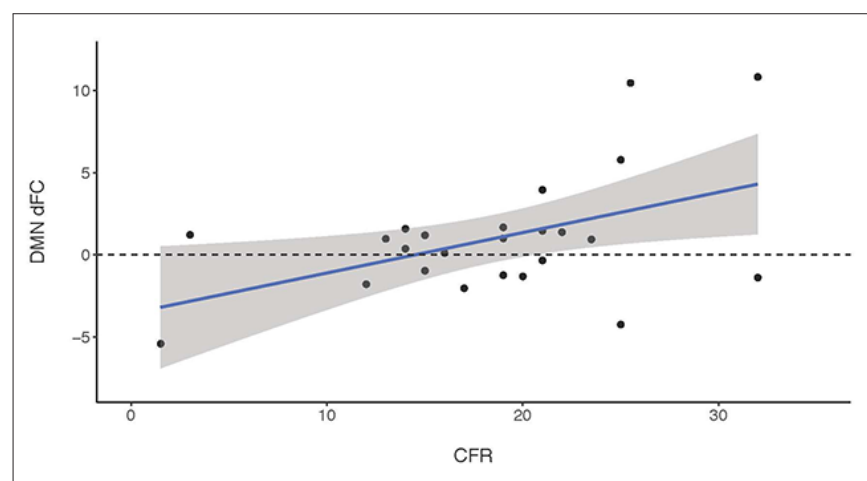

FIGURE 3 | A positive association was found between dFC of the DMN and performance on the visuospatial memory task (Complex Figure of Rey, CFR) in parkinson's disease (PD) patients.

\section{Association Between Dynamic Connectivity and Other PD Symptoms}

A linear hierarchical regression using a forward method was also performed with motor severity and pain intensity as outcome variables. Again, average movement during the scan was added in the first block to control for the possible confounding effect of motion. dFC of the DMN and FPN was not significantly associated with either motor severity or with pain intensity during the OFF phase. See Table 4 for details.

\section{DISCUSSION}

The main aim of this study was to investigate a link between cognitive performance and dynamics of the DMN and FPN in PD patients. First, we found that both dFC of the DMN and dFC of the FPN with the rest of the brain did not significantly differ between PD patients and controls. In addition, we report dFC of the DMN with the rest of the brain as a correlate of visuospatial memory in our patient group.

The positive association between $\mathrm{dFC}$ and visuospatial memory in PD strengthens the findings of a previous study investigating cognitive functioning in $\mathrm{PD}$, also using a sliding window $\mathrm{dFC}$ technique: higher dynamics within the dorsal attention network at rest was found to be predictive for attention performance (Madhyastha et al., 2015a). The association between cognition and dFC has also been investigated in other patient populations: in a group of epilepsy patients, lower dFC of the PCC, a key player in the DMN, with the rest of the brain was related to disturbed verbal memory functioning (Douw et al., 2015), suggesting a similar association between $\mathrm{dFC}$ and memory functioning as was found in our PD group.

Jones and colleagues (Jones et al., 2012) have suggested before that $\mathrm{dFC}$ of the DMN might underlie the cognitive deterioration in people at risk for developing Alzheimer's disease (AD) patients. Our results established a lower dFC of the DMN with the rest of the brain for patients with MCI. These results hint

TABLE 4 | Association between dFC, pain and motor symptoms.

\begin{tabular}{lllcccc}
\hline & & Unstandardized B & Std. error of B & Standardized B & $\boldsymbol{p}$-value & Effect size $\left(\boldsymbol{R}^{\mathbf{2}}\right)$ \\
\hline DMN & Motor severity: & UPDRS III & -0.301 & 0.510 & -0.137 & 0.562 \\
& Pain experience: & NRS & -0.121 & 0.140 & -0.198 & 0.016 \\
FPN & Motor severity: & UPDRS III & 0.239 & 0.250 & 0.216 & 0.398 \\
& Pain experience: & NRS & 0.009 & 0.071 & 0.031 & 0.034 \\
\end{tabular}

Linear hierarchical regression analyses were performed with a forward stepwise method, per symptom. 
that Jones' hypothesis could also hold for PD, and that $\mathrm{dFC}$ of the DMN with the rest of the brain is linked to cognitive fitness, or to the preservation of cognitive functioning.

We found no group differences in $\mathrm{dFC}$ of either network (i.e., DMN or FPN), even though patients' cognitive functioning was worse on several tests. This could be due to methodological choices, such as our focus on dFC of the entire FPN and DMN. Consideration of further subdivisions of each network (e.g., posterior DMN vs. anterior DMN) may increase sensitivity to group differences. This is evident in a study of people with autism spectrum disorder, which showed dFC increases in certain subsets of the DMN and decreases in other subsets of the DMN when compared to controls (de Lacy et al., 2017). Another example is the study of Jones and colleagues (Jones et al., 2016), where dwell time in several subnetworks of the DMN was calculated for a group of AD patients, using a sliding window technique for resting-state connectivity. $\mathrm{AD}$ patients spent more time in the anterior subnetwork of the DMN, but less time in the posterior subnetwork of the DMN. Thus, focusing on different subnetworks of the DMN might improve specificity of conclusions on group differences.

Our second aim was to test whether dFC was specific for certain aspects of cognitive functioning, or whether it might also be related to motor symptoms or pain experience. No association was found between $\mathrm{dFC}$ and motor symptoms, or $\mathrm{dFC}$ and pain. This does not rule out the possibility of an association between $\mathrm{dFC}$ and other symptoms of PD: in the recent study by Kim et al. (2017), severity of motor symptoms was related to time spent in a specific configuration of sparse interconnectedness, which was calculated using a sliding window technique. However, our results suggest that cognition is the main correlate of $\mathrm{dFC}$ of the DMN specifically. To our knowledge, however, no study has investigated a link between $\mathrm{dFC}$ and pain in $\mathrm{PD}$. More research is needed to investigate to what extent $\mathrm{dFC}$ might be specifically underlying cognitive functioning as opposed to other symptoms of PD. Future studies could, for example, select patients on motor-subtypes, such as tremor, akinesia, rigidity or loss of balance.

The results of our study indicate that the $\mathrm{dFC}$ of the $\mathrm{DMN}$ is related to visuospatial memory in PD patients. This association between $\mathrm{dFC}$ and cognitive functioning is not only interesting from a clinical point of view, but it also strengthens the notion that dynamic connectivity is linked to brain function (Hutchison et al., 2013; Sizemore and Bassett, 2017). However, some limitations apply. First, the number

\section{REFERENCES}

Amboni, M., Tessitore, A., Esposito, F., Santangelo, G., Picillo, M., Vitale, C., et al. (2015). Resting-state functional connectivity associated with mild cognitive impairment in Parkinson's disease. J. Neurol. 262, 425-434. doi: 10.1007/s00415-014-7591-5

Baggio, H. C., Segura, B., Sala-Llonch, R., Marti, M. J., Valldeoriola, F., Compta, Y., et al. (2015). Cognitive impairment and resting-state network connectivity in Parkinson's disease. Hum. Brain Mapp. 36, 199-212. doi: 10.1002/hbm.22622

Beck, A., Steer, R., and Brown, G. (1996). Beck depression inventory. Available online at: https://www.nctsn.org/measures/beck-depression-inventorysecond-edition [Accessed on May 15, 2017]. of subjects included in this study is small, and so results cannot be generalized to the entire population of PD patients. Given the heterogeneity of symptoms and symptom severity in the population of $\mathrm{PD}$ patients together with the relatively mild symptoms present in our small sample, one should be careful in extrapolating these results to the entire population of PD patients. Second, factors such as long-term effects of medication and disease duration were not taken into account. Third, cognitive functioning was tested in the ON phase, whereas resting-state data came from the OFF phase. A stronger link might be found if neuropsychological testing would occur in the same medication state as the resting-state scan. Fourth, one should take into account that dynamic connectivity has been introduced quite recently, and its biological correlates are not yet firmly established. Additionally, we report only a correlation between dynamics and cognition in this study. Longitudinal studies may elucidate whether a decrease of dFC precedes cognitive deterioration in $\mathrm{PD}$ or vice versa. Animal and intervention studies, such as pharmacological and brain stimulation studies, could provide evidence for a causal link between brain dynamics and cognitive functions, and determine its exact implications.

\section{CONCLUSION}

This cross-sectional study reports $\mathrm{dFC}$ as a neural correlate of cognitive functioning in $\mathrm{PD}$ : $\mathrm{dFC}$ of the $\mathrm{DMN}$ with the rest of the brain was associated with better visuospatial memory functioning. This association was not found when motor symptoms or pain were considered, which suggests that $\mathrm{dFC}$ of the DMN may be specifically linked to cognitive functioning. This study adds to the understanding of which factors possibly contribute to cognitive functioning in PD.

\section{AUTHOR CONTRIBUTIONS}

GE, BM, AV and ES conceived and designed the experiments. GE and $\mathrm{BM}$ performed the experiments. GE, BM and $\mathrm{LD}$ analyzed the data. GE, BM, AV, ES and LD wrote the article.

\section{FUNDING}

This research was supported by Stichting Parkinson Fonds, Arnold Oosterbaan Hersenstichting, and by the European Research Council Advanced Grant ERC-2012-AdG-323413.

Biundo, R., Weis, L., Facchini, S., Formento-Dojot, P., Vallelunga, A., Pilleri, M., et al. (2014). Cognitive profiling of Parkinson disease patients with mild cognitive impairment and dementia. Parkinsonism Relat. Disord. 20, 394-399. doi: 10.1016/j.parkreldis.2014.01.009

Broen, M. P. G., Braaksma, M. M., Patijn, J., and Weber, W. E. J. (2012). Prevalence of pain in Parkinson's disease: a systematic review using the modified QUADAS tool. Mov. Disord. 27, 480-484. doi: 10.1002/mds. 24054

Broyd, S. J., Demanuele, C., Debener, S., Helps, S. K., James, C. J., and SonugaBarke, E. J. S. (2009). Default-mode brain dysfunction in mental disorders: a systematic review. Neurosci. Biobehav. Rev. 33, 279-296. doi: 10.1016/j. neubiorev.2008.09.002 
Choi, S.-M., Kim, B. C., Jung, H.-J., Yoon, G.-J., Kang, K. W., Choi, K.-H., et al. (2017). Impact of pain and pain subtypes on the quality of life of patients with Parkinson's disease. J. Clin. Neurosci. 45, 105-109. doi: 10.1016/j.jocn.2017. 08.002

Cole, M. W., Reynolds, J. R., Power, J. D., Repovs, G., Anticevic, A., and Braver, T. S. (2013). Multi-task connectivity reveals flexible hubs for adaptive task control. Nat. Neurosci. 16, 1348-1355. doi: 10.1038/ nn. 3470

de Lacy, N., Doherty, D., King, B. H., Rachakonda, S., and Calhoun, V. D. (2017). Disruption to control network function correlates with altered dynamic connectivity in the wider autism spectrum. Neuroimage Clin. 15, 513-524. doi: 10.1016/j.nicl.2017.05.024

Defazio, G., Antonini, A., Tinazzi, M., Gigante, A. F., Pietracupa, S., Pellicciari, R., et al. (2017). Relationship between pain and motor and non-motor symptoms in Parkinson's disease. Eur. J. Neurol. 24, 974-980. doi: 10.1111/ene. 13323

Douw, L., Leveroni, C. L., Tanaka, N., Emerton, B. C., Cole, A. C., Reinsberger, C., et al. (2015). Loss of resting-state posterior cingulate flexibility is associated with memory disturbance in left temporal lobe epilepsy. PLoS One 10:e131209. doi: 10.1371/journal.pone.0131209

Douw, L., Schoonheim, M. M., Landi, D., van der Meer, M. L., Geurts, J. J. G., Reijneveld, J. C., et al. (2011). Cognition is related to resting-state smallworld network topology: an magnetoencephalographic study. Neuroscience 175, 169-177. doi: 10.1016/j.neuroscience.2010.11.039

Douw, L., Wakeman, D. G., Tanaka, N., Liu, H., and Stufflebeam, S. M. (2016). State-dependent variability of dynamic functional connectivity between frontoparietal and default networks relates to cognitive flexibility. Neuroscience 339, 12-21. doi: 10.1016/j.neuroscience.2016.09.034

Hawellek, D. J., Hipp, J. F., Lewis, C. M., Corbetta, M., and Engel, A. K. (2011). Increased functional connectivity indicates the severity of cognitive impairment in multiple sclerosis. Proc. Natl. Acad. Sci. US A 108, 19066-19071. doi: 10.1073/pnas.1110024108

Hochberg, Y., and Benjamini, Y. (1995). Controlling the false discovery rate: a practical and powerful approach to multiple testing. J. R. Stat. Soc. Ser. B 57, 289-300.

Hoops, S., Nazem, S., Siderowf, A. D., Duda, J. E., Xie, S. X., Stern, M. B., et al. (2009). Validity of the MoCA and MMSE in the detection of MCI and dementia in Parkinson disease. Neurology 73, 1738-1745. doi: 10.1212/WNL. 0b013e3181c34b47

Hutchison, R. M., Womelsdorf, T., Allen, E. A., Bandettini, P. A., Calhoun, V. D., Corbetta, M., et al. (2013). Dynamic functional connectivity: promise, issues and interpretations. Neuroimage 80, 360-378. doi: 10.1016/j.neuroimage.2013. 05.079

Jenkinson, M., Beckmann, C., and Behrens, T. (2012). Fsl. Neuroimage. Available online at: http://www.sciencedirect.com/science/article/pii/S105381 1911010603 [Accessed on June 9, 2017].

Jia, H., Hu, X., and Deshpande, G. (2014). Behavioral relevance of the dynamics of the functional brain connectome. Brain Connect. 4, 741-759. doi: 10.1089/brain.2014.0300

Jones, D. T., Knopman, D. S., Gunter, J. L., Graff-Radford, J., Vemuri, P., Boeve, B. F., et al. (2016). Cascading network failure across the Alzheimer's disease spectrum. Brain 139, 547-562. doi: 10.1093/brain/awv338

Jones, D. T., Vemuri, P., Murphy, M. C., Gunter, J. L., Senjem, M. L., Machulda, M. M., et al. (2012). Non-stationarity in the "resting brain's" modular architecture. PLoS One 7:e39731. doi: 10.1371/journal.pone.00 39731

Kim, J., Criaud, M., Cho, S. S., Díez-Cirarda, M., Mihaescu, A., Coakeley, S., et al. (2017). Abnormal intrinsic brain functional network dynamics in Parkinson's disease. Brain 140, 2955-2967. doi: 10.1093/brain/awx233

Kucyi, A., and Davis, K. D. (2014). Dynamic functional connectivity of the default mode network tracks daydreaming. Neuroimage 100, 471-480. doi: 10.1016/j. neuroimage.2014.06.044

Kucyi, A., and Davis, K. D. (2015). The dynamic pain connectome. Trends Neurosci. 38, 86-95. doi: 10.1016/j.tins.2014.11.006

Kucyi, A., Salomons, T. V., and Davis, K. D. (2013). Mind wandering away from pain dynamically engages antinociceptive and default mode brain networks. Proc. Natl. Acad. Sci. U S A 110, 18692-18697. doi: 10.1073/pnas.13129 02110
Lansbergen, M. M., Kenemans, J. L., and van Engeland, H. (2007). Stroop interference and attention-deficit/hyperactivity disorder: a review and meta-analysis. Neuropsychology 21, 251-262. doi: 10.1037/0894-4105.21. 2.251

Litvan, I., Goldman, J. G., Tröster, A. I., Schmand, B. A., Weintraub, D., Petersen, R. C., et al. (2012). Diangostic criteria for mild cognitive impairment in Parkinson's disease: Movement disorder society task force guidelines. Mov. Disord. 27, 349-356. doi: 10.1002/mds.24893

Lynall, M.-E., Bassett, D. S., Kerwin, R., McKenna, P. J., Kitzbichler, M., Muller, U., et al. (2010). Functional connectivity and brain networks in schizophrenia. J. Neurosci. 30, 9477-9487. doi: 10.1523/JNEUROSCI.033310.2010

Madhyastha, T. M., Askren, M. K., Boord, P., and Grabowski, T. J. (2015a). Dynamic connectivity at rest predicts attention task performance. Brain Connect. 5, 45-59. doi: 10.1089/brain.2014.0248

Madhyastha, T. M., Askren, M. K., Zhang, J., Leverenz, J. B., Montine, T. J., and Grabowski, T. J. (2015b). Group comparison of spatiotemporal dynamics of intrinsic networks in Parkinson's disease. Brain 138, 2672-2686. doi: 10.1093/brain/awv189

Martinez-Martin, P., Rodriguez-Blazquez, C., Kurtis, M. M., Chaudhuri, K. R., and Group, N. V. (2011). The impact of non-motor symptoms on health-related quality of life of patients with Parkinson's disease. Mov. Disord. 26, 399-406. doi: $10.1002 / \mathrm{mds} .23462$

Meijer, K. A., Eijlers, A. J. C., Douw, L., Uitdehaag, B. M. J., Barkhof, F., and Geurts, J. J. G. (2017). Increased connectivity of hub networks and cognitive impairment in multiple sclerosis. Neurology 88, 2107-2114. doi: 10.1212/WNL. 0000000000003982

Nasreddine, Z., Phillips, N., Bédirian, V., Charbonneau, S., Whitehead, V., and Collin, I. (2005). The montreal cognitive assessment, MoCA: a brief screening tool for mild cognitive impairment. J. Am. Geriatr. Soc. 53, 695-699. doi: 10.1111/j.1532-5415.2005.53221.x

Olde Dubbelink, K., Hillebrand, A., Twisk, J. W., Deijen, J. B., Stoffers, D., Schmand, B. A., et al. (2014). Predicting dementia in Parkinson disease by combining neurophysiologic and cognitive markers. Neurology 82, 263-270. doi: 10.1212/WNL.0000000000000034

Osterrieth, P. (1941). Le test de copie d'une figure complexe: contribution à l'étude de la perception et de la mémoire. Arch. Psychol. Available online at: http://psycnet.apa.org/record-001 [Accessed on May 18, 2018].

Park, H.-J., and Friston, K. (2013). Structural and functional brain networks: from connections to cognition. Science 342:1238411. doi: 10.1126/science.12 38411

Polli, A., Weis, L., Biundo, R., Thacker, M., Turolla, A., Koutsikos, K., et al. (2016). Anatomical and functional correlates of persistent pain in Parkinson's disease. Mov. Disord. 31, 1854-1864. doi: 10.1002/mds.26826

Postuma, R. B., Berg, D., Stern, M., Poewe, W., Olanow, C. W., Oertel, W., et al. (2015). MDS clinical diagnostic criteria for Parkinson's disease. Mov. Disord. 30, 1591-1601. doi: 10.1002/mds.26424

Power, J. D., Cohen, A. L., Nelson, S. M., Wig, G. S., and Barnes, K. A. (2011). Functional network organization of the human brain. Neuron 72, 665-678. doi: 10.1016/j.neuron.2011.09.006

Putcha, D., Ross, R. S., Cronin-Golomb, A., Janes, A. C., and Stern, C. E. (2016). Salience and default mode network coupling predicts cognition in aging and Parkinson's disease. J. Int. Neuropsychol. Soc. 22, 205-215. doi: 10.1017/s1355617715000892

Saan, R. J., and Deelman, B. G. (1986). De 15-Woordentest A en B (Een Voorlopige Handleiding). Groningen: Afdeling Neuropsychologie AZG.

Sizemore, A. E., and Bassett, D. S. (2017). Dynamic graph metrics: tutorial, toolbox and tale. Neuroimage 180, 417-427. doi: 10.1016/j.neuroimage.2017. 06.081

Sporns, O. (2013). Network attributes for segregation and integration in the human brain. Curr. Opin. Neurobiol. 23, 162-171. doi: 10.1016/j.conb.2012. 11.015

Stam, C. J. (2014). Modern network science of neurological disorders. Nat. Rev. Neurosci. 15, 683-695. doi: 10.1038/nrn3801

Stroop, J. R. (1935). Studies of interference in serial verbal reactions. J. Exp. Psychol. 18, 643-662. doi: 10.1037/h0054651

van Geest, Q., Hulst, H. E., Meijer, K. A., Hoyng, L., Geurts, J. J. G., and Douw, L. (2018). The importance of hippocampal dynamic connectivity in 
explaining memory function in multiple sclerosis. Brain Behav. 8:e00954. doi: $10.1002 /$ brb3.954

Verhage, F. (1964). Intelligentie en leeftijd: Onderzoek bij Nederlanders van twaalf tot zevenenzeventig jaar. (Assen: Van Gorcum). Available online at: http://library.wur.nl/WebQuery/clc/474508 [Accessed June 9, 2017].

Wechsler, D. (1955). Manual for the Wechsler Adult Intelligence Scale. Oxford, England: Psychological Corp.

Wilson, B. A., Evans, J. J., Emslie, H., Alderman, N., and Burgess, P. (1998). The development of an ecologically valid test for assessing patients with a dysexecutive syndrome. Neuropsychol. Rehabil. 8, 213-228. doi: 10.1080/713755570

Xia, M., Wang, J., and He, Y. (2013). BrainNet Viewer: a network visualization tool for human brain connectomics. PLoS One 8:e68910. doi: 10.1371/journal.pone.0068910
Zanto, T., and Gazzaley, A. (2013). Fronto-parietal network: flexible hub of cognitive control. Trends Cogn. Sci. 17, 602-603. doi: 10.1016/j.tics.2013.10.001

Conflict of Interest Statement: The authors declare that the research was conducted in the absence of any commercial or financial relationships that could be construed as a potential conflict of interest.

Copyright (C) 2018 Engels, Vlaar, McCoy, Scherder and Douw. This is an open-access article distributed under the terms of the Creative Commons Attribution License (CC BY). The use, distribution or reproduction in other forums is permitted, provided the original author(s) and the copyright owner(s) are credited and that the original publication in this journal is cited, in accordance with accepted academic practice. No use, distribution or reproduction is permitted which does not comply with these terms. 